\title{
Decomposition Method for Complex 3D Problems in
}

\section{Electron Optics}

\author{
Valentin Ivanov \\ Institute of Computational Technologies SB RAS. 6, Acad. Lavrentiev Prosp., Novosibirsk 630090, Russia
}

\begin{abstract}
The decomposition method was successfully used in solving of 3D problems with complex geometry shape in electron optics for the FDM (Finite Difference Method) and FEM (Finite Element Method) mostly to implement fast and robust parallel algorithms and computer codes. We suggest a new version of similar approach for the BEM (Boundary Element Method) based on the alternating method by Schwartz. This approach substantially reduce the dimension of dense global matrix of algebraic system produced by BEM algorithm to solve a complex problem on as single CPU (Central Processor Unit) desktop computer. New algorithm is iterative one, but exponential convergence for the Schwartz's algorithm creates the fast numerical procedures. We describe the results of numerical simulation for a multi electrode ion transport system. The algorithms were implemented in the computer code "POISSON-3".
\end{abstract}

Key words: Electron optics, decomposition method, numerical-analytical technique, boundary element method.

\section{Introduction}

Numerical simulation of electron optic devices includes evaluation of electromagnetic fields and parameters of trajectories for the charged particles. Field evaluation is the hardest problem when the simulation is provided for complex-shape three dimensional problems. It is reduced to the solving of PDE (Partial Differential Equation) for the Poisson's equation or for the Maxwell's system.

Decomposition procedure is used mostly in solving of PDE in parallelization or when the whole problem dimension exceeds the available computer on-line storage. Fig. 1 gives an example of such problem for the section of linear collider. The section includes a chain of 65 cavities and two couplers for input and output RF (radio frequency) power. The internal structure of the cavities and partitioned mesh are presented at Figs. 2 and 3. The goal of simulation was study of dark currents in accelerator structure [1, 2], when the cold emission from the walls at high field gradients further produces the cascades of secondary

Corresponding author: Valentin Ivanov, Dr., research field: computational physics. E-mail: Vivanov.48@mail.ru.. electrons shown at Fig. 4. The parallel FEM Maxwell's solvers "Omega3p" (frequency domain) and "Tau3p" (time domain) were used for the field evaluation, and the code "Track3p" for a particle tracking. Total number of degree of freedom in those simulations was $10^{7}-10^{8}$, and number of macro particles was few millions.

FEM (Finite Element Method) is a very popular method for the field evaluation in electromagnetism, but there are precision problems of the image electron optics, where BEM (Boundary Element Method) has some advantages. Those problems are solving with the aberration approach which requires computation of high-order derivatives for the field. The main disadvantage for the BEM comparing the FEM is that the BEM approximation generates the dense square matrix whereas FEM produces sparse bandwidth matrix. The solving of linear algebraic problem has the complexity $N^{3}$, but similar problem with sparse matrix requires $N \log N$ operations to get the solution. This limitation is a strong one for simulations of complex 3D (three dimensional) problems based on use the BEM numerical technique. Normally the matrix dimension for direct solving this case is about 


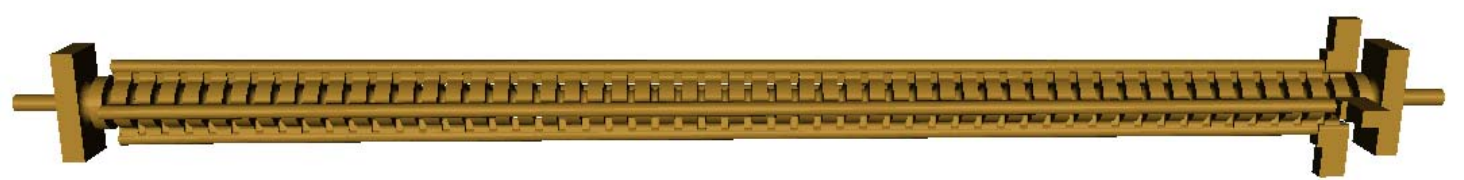

Fig. 1 Large size 65-cell 60-cm X-band travelling wave structure for the Next Linear Collider.
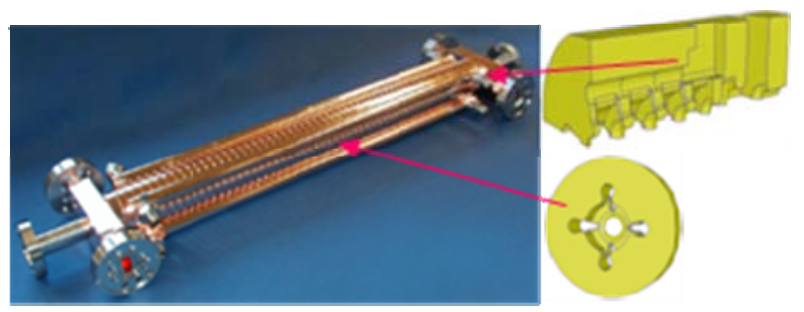

Fig. 2 H60VG4 damped detuned structure.

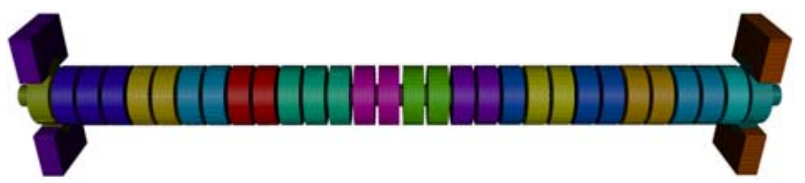

Fig. 3 The partitioned mesh. Different colors correspond to different CPU's.

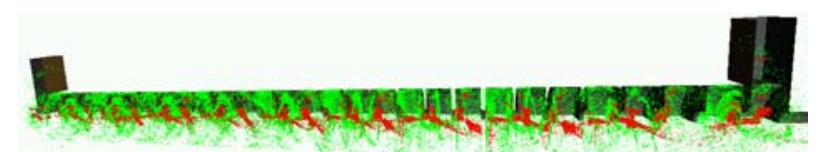

Fig. 4 Dark current simulation for primary particles (red) and secondary ones (green).

$N=2000$. For higher dimensions, the iterative methods are recommended, but study for the stability range and for the convergence rate are needed.

We will describe below the domain decomposition method which has much higher efficiency for such case than the direct solution or iterative procedures for the linear algebraic problems with dense matrices.

\section{Algorithm of the Alternating Method by Schwartz and the BEM Technique}

The BEM approach uses the integral representation for a scalar potential $\varphi$ of electric field

$$
\varphi(r)=\frac{1}{4 \pi \varepsilon_{0}}\left(\int_{S} \sigma\left(s^{\prime}\right) G\left(r, s^{\prime}\right) d s^{\prime}+\int_{V} \rho\left(s^{\prime}\right) G\left(r, r^{\prime}\right) d V^{\prime}\right), s^{\prime} \in S, r^{\prime} \in V(1)
$$

where $r$ - is an observation point, $\varepsilon_{0}$ - is a vacuum permittivity, $\sigma$ - is a surface charge density, but $\rho$ - is a space charge density. The Green's function $G=1 / R$ in 3D case, where $R$ - is a distance between an observation point $r$ and a source points $s^{\prime}$.
Integral representation (Eq. (1)) has an important advantage in aberration approach for the electron optic problems. It has a possibility to evaluate the arbitrary-order derivatives with no accuracy loss as the Green's function $G$ is an analytical one.

Applying the Dirichlet boundary condition

$$
\left.\varphi(r)\right|_{S}=U(r)
$$

with the given function $U$ on the boundary surface $S$, we get the integral equation for unknown function $\sigma$. By similar way we can get corresponding equations for other types of boundary conditions.

Then we represent the surface $S$ with parametric equations

$$
S=\bigcup_{i=1}^{n} S_{i}, x=x_{i}(\tau, \eta), y=y_{i}(\tau, \eta), z=z_{i}(\tau, \eta)
$$

and use some approximations for the surface charge density $\sigma$ and space charge density $\rho$. For example, linear approximations could be written as

$$
\begin{gathered}
\sigma(\tau, \eta)=\left\{\left[\sigma_{i+1, j+1}\left(\tau-\tau_{i}\right)+\sigma_{i, j+1}\left(\tau_{i+1}-\tau\right)\right]\left(\eta-\eta_{j}\right)+\right. \\
\left.\left[\sigma_{i+1, j}\left(\tau-\tau_{i}\right)+\sigma_{i, j}\left(\tau_{i+1}-\tau\right)\right]\left(\eta_{j+1}-\eta\right)\right\} / h_{i i} h_{\eta j}, \\
h_{\tau i}=\left(\tau_{i+1}-\tau_{i}\right), h_{\eta j}=\left(\eta_{j+1}-\eta_{j}\right)
\end{gathered}
$$

and

$$
\begin{aligned}
& \rho(x, y, z)=\left\{\left[\left(\rho_{i+1, j, k}\left(x-x_{i}\right)+\rho_{i, j, k}\left(x_{i+1}-x\right)\right)\left(y_{j+1}-y\right)+\right.\right. \\
& \left.\left(\rho_{i+1, j, k}\left(x-x_{i}\right)+\rho_{i, j, k}\left(x_{i+1}-x\right)\right)\left(y-y_{j}\right)\right]\left(z_{k+1}-z\right)+ \\
& \quad\left[\left(\rho_{i+1, j, k+1}\left(x-x_{i}\right)+\rho_{i, j, k+1}\left(x_{i+1}-x\right)\right)\left(y_{j+1}-y\right)+\right. \\
& \left.\left.\quad\left(\rho_{i+1, j, k+1}\left(x-x_{i}\right)+\rho_{i, j, k+1}\left(x_{i+1}-x\right)\right)\left(y-y_{j}\right)\right]\left(z-z_{k}\right)\right\} / \\
& \quad\left\{\left(x_{i+1}-x_{i}\right)\left(y_{j+1}-y_{j}\right)\left(z_{k+1}-z_{k}\right)\right] .
\end{aligned}
$$

Now we should replace the integrals over the surface $S$ and volume $V$ with some numerical quadratures, and finally we get the linear algebraic system $\hat{G} X=F$ with the global matrix $\hat{G}$, unknown vector $X$ and right-hand vector $F$. The analytical technique is intensively used in numerical solving of 
the integral equations [3, 4]. The efficiency of this technique was demonstrated in simulations of sheet-beam guns for the high power klystrons $[5,6]$.

The CPU-time $T$ for the boundary problem solving can be represented with the formula $T=a N^{3}+b N^{2}$, where $N$ - is the dimension of matrix $\hat{G}$, but $a$ and $b$ are some numerical constants. First term of this formula is a time for solving of the linear system by the Gauss elimination method, and second term is a time for evaluation of the matrix elements. First term dominates for large enough $\mathrm{N}$.

To overcome this strong cubic dependence, H.Schwarts [7] suggested the decomposition idea, when a large problem is spitted onto $K$, overlapped blocks separated each other with the auxiliary boundaries (Fig. 5). Then $K$ boundary problems of lower dimensions are solving in the iterative loop, where the boundary condition on the auxiliary bounds are evaluated from the solution of neighbor block at previous iteration

$$
\left.U_{k}^{(i)}\right|_{n}=\varphi_{k+1}^{(i-1)},\left.U_{k}^{(i)}\right|_{p}=\varphi_{k-1}^{(i-1)}
$$

Here $i$ - is an iteration number, $k$ - is a block number, $U_{k}$ - is a boundary condition for the block $k$ evaluated at the auxiliary boundary from the solution $\varphi$ for the neighbor block at previous iteration. Index $n$ means the boundary with the "next" block, but index $p$ means the boundary with the "previous" block. The boundary conditions for auxiliary bounds at fist iteration can be determined by an arbitrary way (for example, by zero values). H. Schwartz proved the exponential convergence $\sim e^{-\lambda}$ of iteration for this numerical scheme, where $\lambda$ is determined by the bandwidth of the overlapped area.

We can make an estimation for the efficiency of decomposition algorithm comparing with the direct solution of the whole problem of dimension $N$. Let in simplest case all blocks have equal dimensions, i.e. $N$ $=K m$. Then the relative efficiency for large $N$ can be estimated by the formula

$$
E=\frac{a N^{3}+b N^{2}}{l K\left(a m^{3}+b m^{2}\right)} \approx \frac{K^{2}}{l}
$$

where $l$ - is a total number of iterations to reach the predefined accuracy $\varepsilon$. We will show below that typical value $l=3 \div 4$ for $\varepsilon=0.1 \%$.

\section{An Example of the Domain Decomposition Numerical Technique}

As an example of practical application, we examine the conveying device with A-wave profile [8], which is an effective tool for the accurate transport the ensemble of charge particles with large mass dispersion. The electric field of this device is described with the pseudo potential

$$
\begin{gathered}
\bar{U}(z / L, t)=\frac{e U_{0}^{2}}{4 m L^{2} \omega^{2}}\left[\Phi_{\cos }^{\prime}(z / L) \cos (t / T)+\right. \\
\Phi_{\sin }^{\prime}(z / L) \sin (t / T)+ \\
\left.\Phi_{3 \cos }^{\prime}(z / L) \cos (3 t / T)+\Phi_{3 \sin }^{\prime}(z / L) \sin (3 t / T)\right]^{2}
\end{gathered}
$$

where

$$
\begin{aligned}
& \Phi_{\cos }(z / L)=\cos (z / L)\left[F^{a}(z / L)+\frac{3}{4} F^{b}(z / L)+\frac{9}{8} F^{c}(z / L)\right], \\
& \Phi_{\sin }(z / L)=\sin (z / L)\left[F^{a}(z / L)+\frac{3}{4} F^{b}(z / L)+\frac{9}{8} F^{c}(z / L)\right], \\
& \Phi_{3 \cos }(z / L)=\cos (3 z / L)\left[-\frac{1}{12} F^{b}(z / L)+\frac{1}{24} F^{c}(z / L)\right], \\
& \Phi_{3 \sin }(z / L)=\sin (3 z / L)\left[-\frac{1}{12} F^{b}(z / L)+\frac{1}{24} F^{c}(z / L)\right] .
\end{aligned}
$$

Here $L$ - is a spatial scaling coefficient, $T$ - is the characteristic time of A-Wave modulation, $\omega-$ is the base frequency of the RF field, $z$ - is the axial coordinate, $t$ - is a time, $U_{0}-$ is the magnitude of applied voltage.

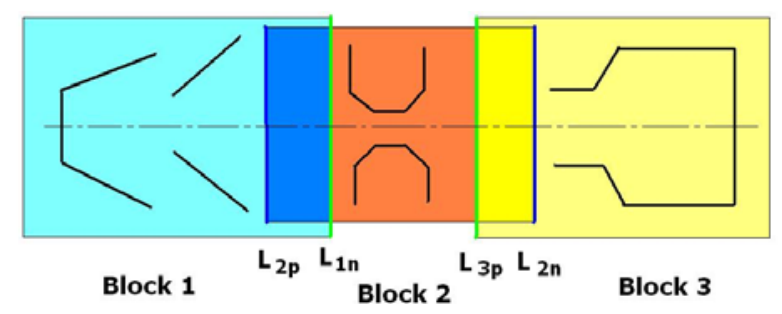

Fig. 5 Domain decomposition scheme. 


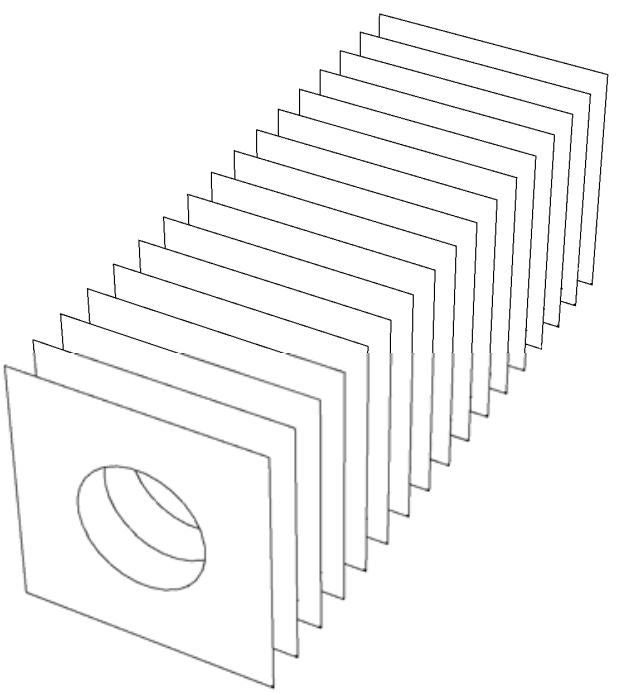

Fig. 6 Stack of electrodes reproduces the A-wave profile.
This field can be reproduced with a stack of planar plates with round orifices (Fig. 6). Some of these plates can have rectangle and triangle holes to correct the quadruple and sextuple aberrations. In numerical simulations, we used the set of parameters: total number of plates $N p=100$, the gaps between plates $s$ $=5 \mathrm{~mm}$, voltage $U_{0}=0.5 \mathrm{kV}$. Two planes of symmetry were used

$$
\varphi(x, y)=\varphi(-x, y)=\varphi(x,-y)=\varphi(-x,-y)
$$

so a quarter of each plate only was used in simulation. Total number of degrees of freedom $N=10^{4}$. The whole stack was divided onto $K=20$ groups with $m=$ 10 plates in each.

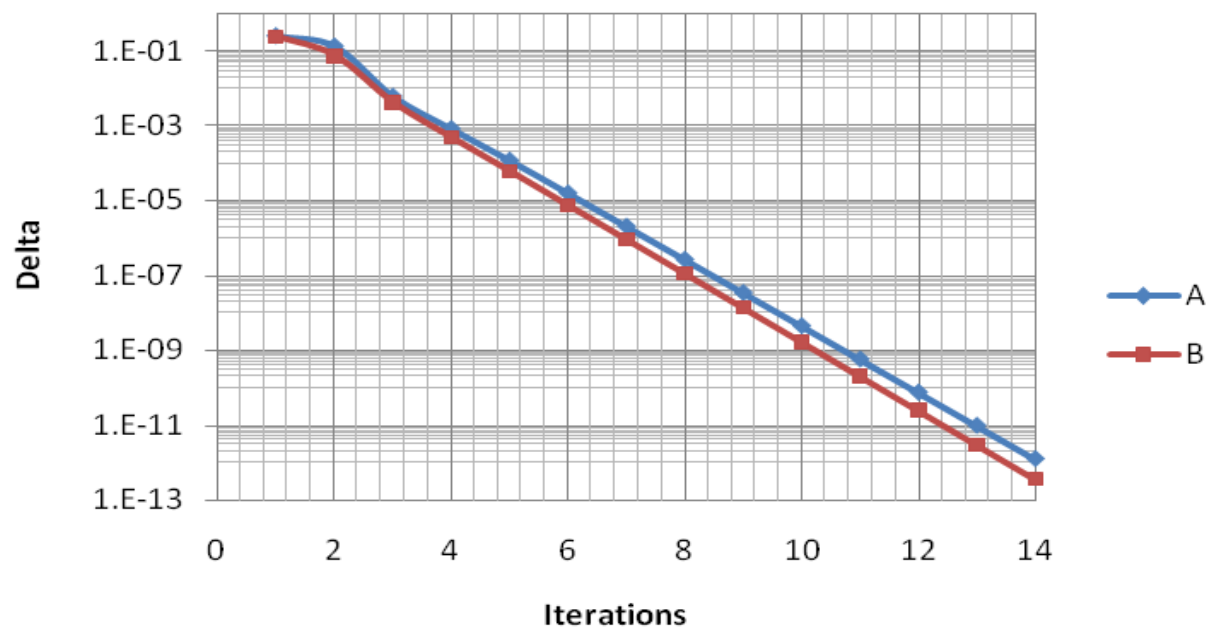

Fig. 7 Convergence of Schwartz iterations. $A-\lambda=0.1, B-\lambda=0.2$.

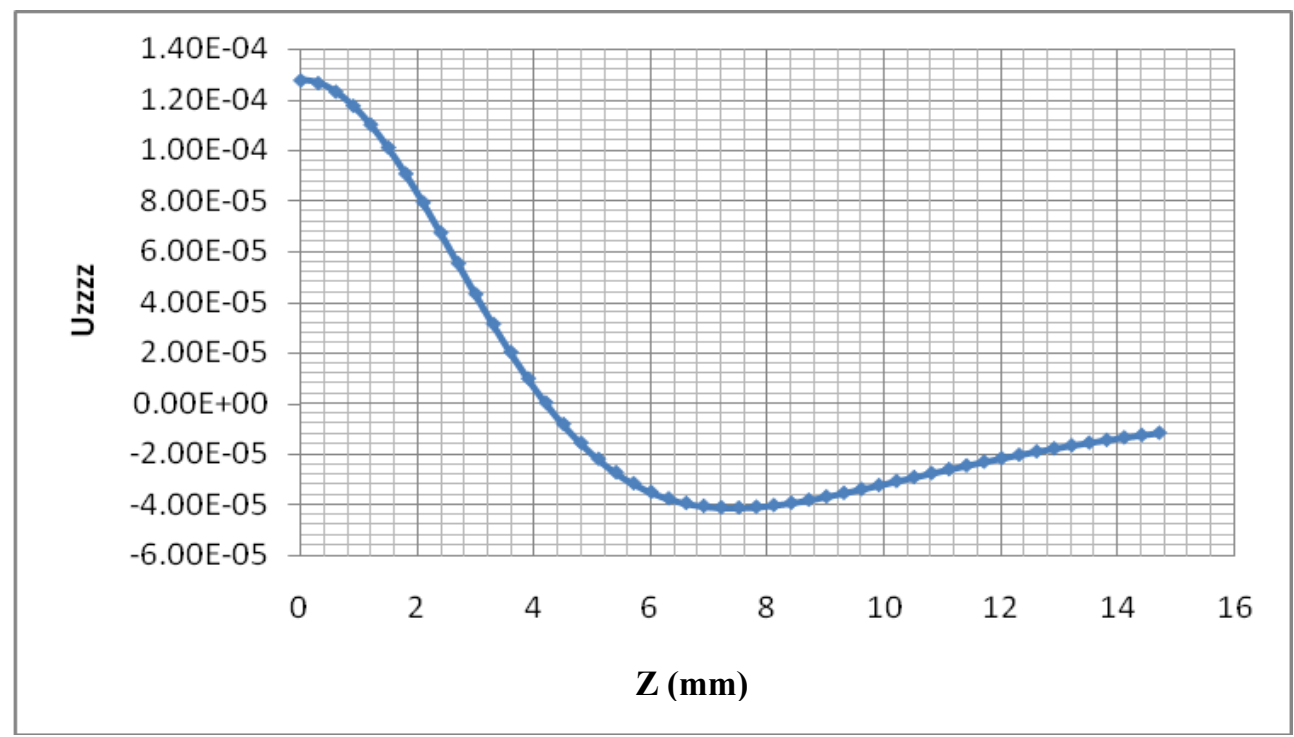

Fig. 8 Axial distribution for the $4^{\text {th }}$-order derivative in the gap between plates. 
Fig 7. shows the convergence of iterations, where the convergence criterion was

$$
\delta=\left\|\sum_{i}\left(\varphi_{i}^{(k)}-\varphi_{i}^{(k-1)}\right)\right\|_{L 2} \leq \varepsilon
$$

where $k$ - is an iteration number, and summation is provided over the index $i$ for all auxiliary bounds. It took $l=4$ iterations for the stop criterion $\delta<\varepsilon=$ $0.01 \%$. The overlapping area for the auxiliary bounds varied between $\lambda=0.1$ (curve A) and $\lambda=0.2$ (curve $B)$. It affects on the convergence rate.

Formula (6) shows the decomposition algorithm works two orders faster than the direct solution for the matrix $N=10^{4}$. Axial distribution for the $4^{\text {th }}$ order derivative $\Phi^{(I V)}(z)$ is shown at Fig. 8 .

\section{Conclusions}

The domain decomposition numerical algorithms and the implemented computer codes demonstrate high accuracy and efficiency in the computer design of various instruments and devices as for self-consistent non linear problems of high-current electronics as for the problems of precision image optics. The efficiency and accuracy of implemented algorithm were studied at the conveying devise with more than hundred conducting electrodes with complex shape holes. Our simulations show high accuracy and relatively modest computation time at desktop computer.

\section{References}

[1] Ivanov, V., Ajolphsen, C., Folwell, N., Ge, L., Guetz, A., Li, Z., and et al. 2003. In Proc. Particle Accelerator Conference (PAC 03), 664-2666.

[2] Li, Z. H., Folwell, N., Ivanov, V., Ge, L. X., and Guetz, A. 2006. Nucl. Instrum. and Meth. A 558: 168-174.

[3] Ivanov, V. 2009. Int. J. of Modern Physics A 24 (5): 869-878.

[4] Ivanov, V. 2014. Nauchnoe Priborostroenie 24 (1): 96-103.

[5] Ivanov, V., Ko, K., Krasnykh, A., Ives, L., and Miram, G. 2001. In Proc. of IEEE Particle Accelerator Conference, 1213-5

[6] Ivanov, V., Krasnykh, A., Read, M. E., Miram, G., and Ives, R. L. 2003. In Proc. of Particle Accelerator Conference, 2562-5.

[7] Schwartz, H. 1890. Gez. Math. Abh. Bd 2 (B): 185-193.

[8] Andreyeva, A. D., and Berdnikov, A. S. 2012. Journal of Analytical Chemistry 67 (13): 1034-7. 